\title{
Buffered-microgrid Structure for Future Power Networks; a Seamless Microgrid Control
}

\author{
Nachat Nasser, Member, IEEE, Meghdad Fazeli, Senior Member, IEEE
}

\begin{abstract}
This paper proposes a new structure and control scheme for future microgrid-based power system, which is designed to achieve a seamless operation in both islanded and gridconnected modes, while the load is appropriately shared by all units (i.e. renewable sources, energy storage systems and the grid). The proposed method, which involves physical separation of the microgrid from the grid by using AC/DC/AC converters, ensures safe, secure and seamless operation of both modes. Such a "buffered" structure enables reduction in the transmission losses by reducing the exchanged energy with the grid through using a dead-zone in the control of the buffering AC/DC/AC converter. An inverse-droop control technique has been implemented to control the voltage magnitude and frequency, using current control in the $d q$-frame. PSCAD/EMTDC software has been used to validate the proposed method through simulating different scenarios. The solution provides a simple, smooth, and communication-free decentralized control for multi-sources microgrids. Moreover, the proposed buffered structure separates the dynamics of the microgrid and the grid, which enables a faster microgrid voltage and frequency control and protects the grid and the microgrid from faults on the other side.
\end{abstract}

Index Terms - Droop Control, Energy Storage, Microgrids, Primary Control, Renewable Energy, Seamless Operation.

\section{INTRODUCTION}

$\mathrm{T}$ He future trend of the electricity sector is firmly linked to the commitments of carbon emission reduction and to the shortage of fossil fuel sources. The UK is committed to reduce the greenhouse gas emission up to $100 \%$ of the 1990 level by 2050 [1], where all future energy scenarios expect up to $58 \%$ of generation to be decentralized and about $75 \%$ of the GB future electricity would come from renewable energy (RE) sources and energy storage (ES) systems by 2050 [2]. The distributed location of RE sources, which led to the creation of the distributed energy resources (DERs), causes several challenges for the network operators to manage the system.

Manuscript received ...; revised ... and ...; accepted ... . Date of publication .. ; date of current version ... . Paper no. ... .

This work is supported by the FLEXIS project, which is part-funded by the European Regional Development Fund through the Welsh Government.

N. Nasser is a Research Officer with the Energy Safety Research institute College of Engineering, Swansea University, UK (e-mail: nachat.nasser@swansea.ac.uk).

M. Fazeli is a Senior Lecturer with the Energy Safety Research institute College of Engineering, Swansea University, UK (e-mail: M.Fazeli@swansea.ac.uk).
Microgrids (MGs), which are clusters of distributed generation (DG), ES and controllable loads, are proposed as a tool to accommodate and manage more DERs [3]-[5]. MGs, which are supposed to operate in both islanded and grid-connected modes, aimed to reduce transmission losses and provide local ancillary services [6], [7]. Different control approaches have been developed to coordinate the power management within an $\mathrm{MG}$, such as the centralized, distributed, peer-to-peer and local control (e.g. droop control) architectures [8]. Some of these methods (e.g. peer-to-peer) rely heavily on communication, which can cause disruption in case of a failure in the communication link. Moreover, most (if not all) commercialized MGs utilize the master-slave scheme in islanded mode, where the ES unit acts as the master unit and provides voltage and frequency for the other units to follow. This approach suffers from lack of redundancy in case of a fault occurs on the master unit. To overcome these drawbacks droop control has been proposed, where the local active and reactive powers are measured and used to set the voltage and frequency according to the droop gains [8]-[10].

Load sharing and ES control have been investigated in several previous arts [11]-[16]. In [11], a power sharing strategy based on droop control and communication platform was introduced. It requires uploading the power output information of the units to a microgrid control centre, and receiving the power sharing instructions to regulate the power flow and voltage of the MG. In [12], which is a communication-free method, an angle droop control with a high droop gain is used to ensure a proper load sharing. However, since the high gain droop control has a negative impact on the overall stability [12], a supplementary loop around the droop control of each DG is used to mitigate the negative impact. Reference [13] proposed a local load sharing technique for distributed MG with local and common loads. It assumed that the common load is supplied solely by the utility in the grid-connected mode, while the load will be shared by the DGs through traditional droop method when an islanding occurs. Hence, the reference voltage is set from the positive sequence fundamental component of the point of common coupling, while load sharing strategy is based on adjusting the droop parameters at each DG through measuring the power differences to generate the control references. Because of having two different control methods for islanded and grid-connected modes, [13]requires an islanding detection scheme. In [14], a dynamic droop scheme for islanded MGs, which is sensitive to the available solar power from units, was 
introduced. It showed that using the proposed method, the DGs compensate for one another, which reduces the contribution from an auxiliary generator within the MG. In [15], the regulation of frequency droop was studied by using the battery state of charge (SoC) to control the power reference in order to regulate the battery charging process and the load sharing. However, the method imposed constraints on the generated power from the photovoltaic (PV) units by controlling its operating point to regulate the power sharing between the load and the battery. Reference [16] introduced a decentralized power management and load sharing method for a PV-based islanded microgrid consisting of PV units, battery units and hybrid PV/battery units. In this method, the whole MG can operate in three modes and the operation of each unit in the MG is divided into five states according to load, PV generation, and battery SoC. The frequency level is used as a trigger for transition between the states, where in each state, a specific modified droop function is used for the output power control.

Another important issue in MGs operation is synchronization, particularly, at the time of reconnection to the grid [17]-[19]. In [17], a tuned controller of virtual-air-gap variable reactor technique was developed to realize $\mathrm{MG}$ reconnection to the grid. Even though this method is useful when a large communication area is not available, it necessitated the usage of a hold period in order to keep the reactance at its maximum value to limit the peak current. Since this hold period is based on the inertia constants of the two interconnected networks and the relative voltage-angle difference between them, a communication is required. The seamless reconnection method proposed in [18] considered both the positive and negative sequences of the harmonic component, in addition to the fundamental one. Beside the parameters limit check, which is required for MG synchronization criteria, a power quality check for the MG and the main grid is required to be within an acceptable limit before closing the static transfer switch. Reference [19] proposed a distributed cooperative control framework to overcome the reconnection synchronization issues of multi-bus MG. It proposed that all the involved DGs should adjust their outputs in order to cooperatively regulate the voltage and frequency of the MG to track those of the main network. Therefore, a sparse communication infrastructure is required to achieve the proposed method.

One common drawback of these methods is that they necessitate an islanding detection algorithm to switch from the grid-connected to islanded mode [20]. To overcome this drawback a number of control approaches have been proposed more recently, e.g. [21]-[23]. Reference [21] proposed a cascaded hybrid frequency-phase angle droop alongside extra power loops, which imply some unnecessary operational complexity. Moreover, a communication interface is required for the secondary control. Authors of [22] proposed a single decentralized control law based on a model for uncertainties (which are estimated by an extended state observer) through a combination of integral control and partial input saturation. This method achieves voltage magnitude and frequency regulation for both islanded and grid connected operation, including seamless transition operation. However, it didn't investigate the response of the system to faults, change of
load/DER's power, and the operation of an ES system. The work introduced in [23] proposed two parallel converters to interface each DG unit in a MG. One of them is controlled in voltage-controlled mode while the other is controlled in current-controlled mode. Although this study showed an enhancement of control dynamics in grid-connected mode and an improved load sharing performance during the islanded operation, it imposed some disadvantages such as the additional costs of using two converters, two controller and the additional measurement units per each DG unit. It also imposed more complexity on the control circuit where sensing the load power and the operation mode is required.

As discussed above, several methods and approaches have been proposed to improve the operation of MGs, but most of them suffer from different disadvantages such as operational constraints [15], control complexity [21], [23], [24] communication interface [17], [19] or additional costs [23]. In addition, previous arts did not consider all scenarios, e.g. [22], [24], despite being a communication-less method, did not investigate short circuits, which can be challenging for an observer-based method [22] and a method that uses a small disturbance signal [24], which might deteriorate during the fault. To overcome the above-mentioned drawbacks, this paper proposes a simple, communication-free droop-based control method, in which, the MG is totally supplied by inverterinterfaced sources.

Moreover, in all previous arts, because of the direct connection between the MGs and the grid, their dynamics and operational conditions (e.g. faults) will affect the other side. For instance, it is reported in [25] that the interaction between reactive power controller of wind generators and synchronous generators (SGs) might generate some new low frequency oscillatory modes. The direct connection between the grid and the MG becomes more problematic during grid re-connections, where care must be taken to make sure that the voltage magnitude, frequency and the phase angle of both sides (i.e. the grid and the MG) are the same prior to the reconnection. Unsynchronized reconnection can lead to sever torsional oscillations which can even break the shaft of SGs. Therefore, SGs have protection systems that trip in case of even a small phase angle difference. Note that adjusting the rotor angle of SGs on the grid side will necessitate communications from the MG to them. On the other hand, adjusting the phase angles of the DERs (of the MG) while they are operating in islanded mode will cause circulating current that may trip them.

To solve this issue, this paper, as shown in Fig. 1, proposes to decouple the MG from the grid by a set of back to back $\mathrm{AC} / \mathrm{DC} / \mathrm{AC}$ converters. The advantages of this solution over other related research can be summarized as follows:

- Requires no communication interface, neither between the MG units, nor with the main grid in all operational modes.

- No power generation constraint is applied to DER units, allowing full exploitation of the available input power, unless the battery is fully charged while the MG is islanded. In such a case, the DER power is, seamlessly, reduced to match the connected load i.e. generation shedding (Fig. 7).

- Applicable to both inductive and resistive loads (Fig. 5). 


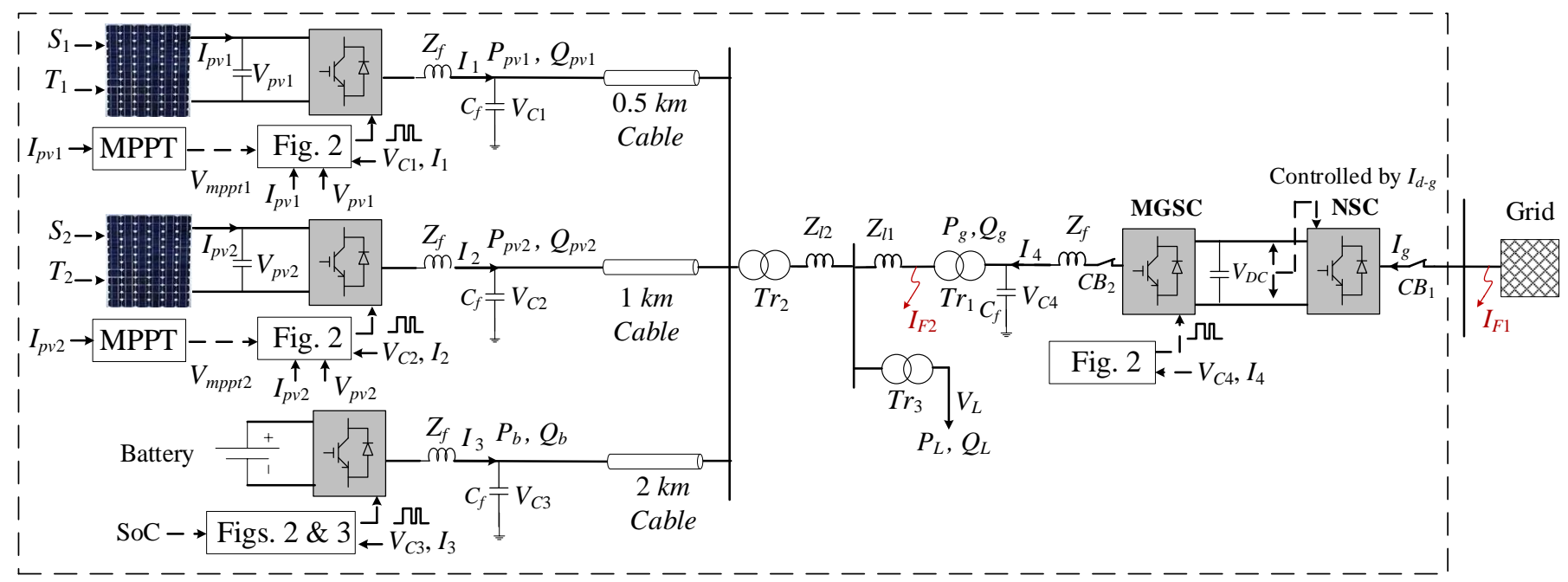

Fig. 1. The system under study.

- Physical isolation between the MG and the grid, which protects the MG against the grid-side disturbances and fault events (Fig. 7). Moreover, this structure protects the MG from the low frequency inter-area oscillations caused by the SGs on the grid.

- $\quad$ Simple industry-standard $d q$-frame-based control (Fig. 2).

- Robustness against large disturbances such as load changes (Figs. $5 \& 7$ ) and DG outage (Figs. $7 \& 10$ ).

- Reducing the energy exchanged with the main grid (by proposing a dead-zone in the control), which reduces the transmission loss (Fig. 6).

- Enabling the coordination between units through a central system. Although this is not the focus of the paper, it is shown (in Fig. 11) that it is possible to override the main controller to coordinate between units if needed (e.g. charging/discharging the ES from/to the grid over nights)."

It is noted that while some of the listed advantages (e.g. physical isolation) are unique to this paper, there are previous works that offer some of the above advantages. However, to the best of the authors' knowledge, there is no previous art that offers all the above advantages.

The proposed control method in this paper is based on [20] where the authors introduced one control paradigm for all operational scenarios of a MG, consisting of a PV-Battery system and an auxiliary generator. The work offered a comprehensive active and reactive power management scheme with maximum power point tracking (MPPT) and ES control. This method keeps the phase locked loop (PLL) circuit part of the control scheme during the islanding to ensure automatic resynchronization when reconnecting to the grid. Since the method proposed in [20] does not require any communication between the grid and the MG, it is chosen as the base for the proposed structure/control in the current paper. The main improvements over [20] are as follows:

- Whereat [20] requires communication within the MG, the current paper proposes a control scheme that makes the energy management totally communication-free for all modes of operation.
- The current paper proposes a dynamic time-constant selection for the virtual governor and the virtual Automatic Voltage Regulator (AVR) that provides superior damping for all types of loads (inductive and resistive) and eliminates frequency oscillations at low loads, without sacrificing the dynamic performance.

- This paper proposes a new battery management system that, in contrast to [20], allows maximum charging/discharging capacity until the maximum/minimum SoC is reached.

- More importantly, this paper proposes a new structure (as well as control), which were not investigated in [20] and offers additional advantages such as protection from external faults and low frequency oscillations.

\section{PROPOSED STRUCTURE AND CONTROL METHOD}

The system under study, shown in Fig. 1, consists of an MG connected to an infinite network through a set of AC/DC/AC converters. The MG consists of two PV systems and a battery ES system, while each of them is interfaced by a VSC, providing $11 \mathrm{kV}$ AC power, to be stepped up through 11/275 $\mathrm{kV}$ power transformer. DC/AC converters, rated at $5 \mathrm{MVA}$, are used to connect the PV and ES units (considering the space limitation, only three units are studied, to be able detailing their control and displaying all the necessary results). The decoupling AC/DC/AC converter, rated at 5 MVA, consists of an MG side converter (MGSC) and a network side converter (NSC). While the MGSC is controlled through the proposed algorithm in this paper (detailed below), the NSC controls the DC link voltage $V_{D C}$ through regulating the grid's directcomponent current $I_{d-g}$. The MG feeds a variable $5 \mathrm{MVA}$ load $\left(P_{L}, Q_{L}\right)$ through a $275 / 0.65 \mathrm{kV}$ power transformer.

The control methods for the PV converter, battery converter and MGSC, which are illustrated in Figs.1 and 2, are very similar in principle, but not identical. As shown, all units are controlled using a rendition of $I_{d^{-}} V, I_{q}-f$ droops, which make the system works for both inductive and resistive loads (see Fig. 5). In principle, the method also works with $I_{d}-f, I_{q^{-}} V$ droops, but only if the network is predominantly inductive. Intuitively speaking, since the transferred energy is proportional to the 


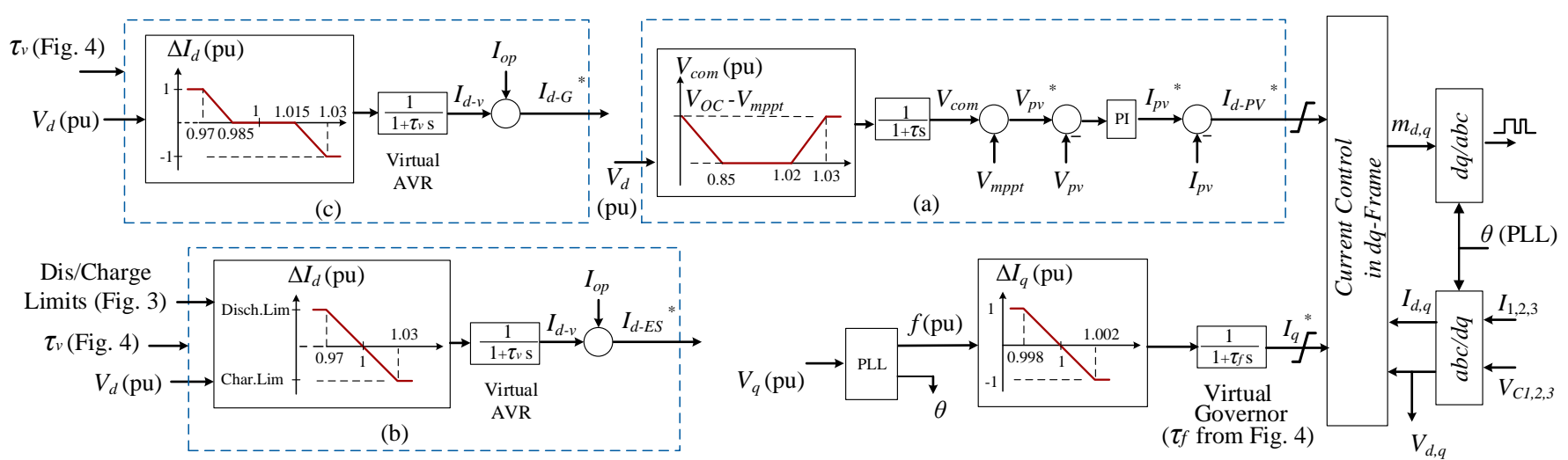

Fig. 2. The control scheme applied to all source units. (a) Active power control used for $P V$ control. (b) $I_{d}-V_{d}$ droop scheme for ES control. (c) $I_{d}-V_{d}$ droop scheme for the MGSC.

potential difference (voltage), using voltage to regulate active power (rate of change of energy) makes sense $\left(I_{d}-V\right.$ droop). Also, since reactive power is due to the phase difference between voltage and current, using frequency (rate of change of the phase angle) to regulate reactive power makes sense $\left(I_{q}-f\right.$ droop). The reason that conventionally active power and frequency are mainly related is the electromechanical coupling between the SGs and the power system. Due to this coupling the active power variations will be reflected on the SGs' speed, which is proportional to the frequency. However, since the buffering converters in Fig. 1 separate the dynamics of the MG from the grid, the SGs' speed on the grid will not affect the MG's frequency. Therefore, it is possible to use $I_{d^{-}} V, I_{q}-f$ droops for all types of load. Note that this is in agreement with the "universal droop" control proposed in [26]. As shown in Figs. $1 \& 2$, local voltages $V_{C_{1,2,3}}$ and local currents $I_{1,2,3}$ are measured and transformed to $d q$-frame $\left(I_{d}, I_{q}, V_{d}, V_{q}\right)$. Each unit has a synchronously-reference-frame PLL that provides the phase angle $\theta$ (for the Park transform) and the local frequency $f$. Note that the PLLs also impose the nominal frequency on each DG and synchronize them through regulating their local $V_{q}=0$. The nominal local voltage is implied during the process of calculating the per unit (pu) value for the local $V_{d}$. All converters are current-controlled VSC using PI controllers.

Each locally measured frequency of PVs, ES and MGSC is fed to its virtual governor, which is identical for all units and provides the reference $q$-component current $I_{q}{ }^{*}[20]$. The virtual governor uses $I_{q}-f$ droop, which is described by (1) [20]:

$$
\Delta I_{q}=K_{f}\left(f-f_{0}\right)
$$

where $K_{f}$ is the droop gain, and $f_{0}=1 \mathrm{pu}$ is the nominal frequency. A frequency deviation range of $\pm 0.2 \%$ is applied (which can be changed according to different Grid Codes). To emulate the damping characteristics of SGs, the output of each $I_{q}-f$ droop is passed through a first-order low-pass filter with time constant $\tau_{f}$.

Unlike virtual governor, which is identical for all types of unit, this paper proposes different active power regulation for PVs, ES and MGSC:

$P V$ systems: Unlike wind turbines, since PV arrays do not have any inherent storage capacity, they cannot participate in inertial services. When there is no large voltage sag nor a voltage rise, the PV system is expected to operate on MPPT (Figs. 1 and 2(a)). The reference PV-DC voltage $V_{p V}^{*}=V_{m p p t}+V_{\text {com }}$, where $V_{m p p t}$ is set by an MPPT algorithm, and the compensation voltage $V_{\text {com }}$ is set by the proposed method, illustrated in Fig. 2 (a) ( $V_{O C}$ is the open circuit voltage of the PV array):

- For $0.85 \leq V_{d} \leq 1.02 \mathrm{pu}, V_{c o m}=0 \rightarrow V_{p V}^{*}=V_{m p p t}$.

- For $0 \leq V_{d}<0.85 \mathrm{pu}, V_{\text {com }}$ increases proportionally up to $V_{O C}-V_{m p p t} \rightarrow V_{p V}^{*}$ increases proportionally up to $V_{O C}$, which reduces $I_{p v}$ and $I_{d-P V}^{*}$. This is a simple Low Voltage Ride Through (LVRT) algorithm.

- For $1.02<V_{d} \leq 1.03 \mathrm{pu}, V_{\text {com }}$ increases proportionally up to $V_{O C}-V_{m p p t} \rightarrow V_{p V}^{*}$ increases proportionally up to $V_{O C}$, which reduces $I_{p v}$ and $I_{d-P V}^{*}$. This is a simple Generation Shedding algorithm, which will only happen in islanded MGs if $P_{L}<$ $P_{P V}$ and the ES is fully charged. Doing this reduces the generation and prevents over voltage on both AC and DC sides of the PV inverter. Moreover, this enables a smooth and seamless transition without any dumping mechanism.

ES and MGSC, in principle, utilize the same $I_{d}-V_{d}$ droop, which is described by (2) [20]:

$$
\Delta I_{d}=K_{v}\left(V_{d}-V_{0}\right)
$$

where $K_{v}$ is the droop gain and $V_{0}=1 \mathrm{pu}$ is the nominal voltage. A first-order low-pass filter, with time constant $\tau_{v}$ is used to emulate the AVR behavior of the SGs [20]. A voltage deviation of $\pm 3 \%$ is considered. The $d$-component voltage $V_{d}$ of ES and MGSC is fed to their virtual AVR (Figs. 2(b) and 2(c)), respectively, which sets $I_{d-v}$ of them. The reference $d$ component current is then calculated as:

$I_{d}{ }^{*}=I_{d-v}+I_{o p}$, where $I_{o p}$ can be used by a centralized system to coordinate between units by overriding the main controller (e.g. to buy/sell energy). The centralized system is not the main concern of this paper, however, a simple scenario is explained in section III.

ES systems: This paper focuses on battery ES systems; however, the principles are applicable to other types of ES as well. $I_{o p}$, which is used only by a centralized system to coordinate between units, is zero during normal operation. The droop characteristics for the virtual AVR, which is illustrated in 
Fig. 2(b), is as follow:

If there is an excess energy:

- For $1<V_{d}<1.03 \mathrm{pu} \rightarrow$ charges proportionally.

- For $V_{d} \geq 1.03 \mathrm{pu} \rightarrow$ charges at maximum limit.

If there is a lack of energy:

- For $0.97<V_{d}<1 \mathrm{pu} \rightarrow$ discharges proportionally.

- For $V_{d} \leq 0.97 \mathrm{pu} \rightarrow$ discharges at maximum limit.

Assuming that the ES is fully charged at $\mathrm{SoC}=90 \%$ and fully discharged at $\mathrm{SoC}=20 \%$ (these can change for different batteries), the charge/discharge limits are set as (see Fig. 3):

- For $21<$ SoC $<89 \%$, charge/discharge limits $=1$ pu.

- For SoC $\geq 90 \%$, charge/discharge limits $=0 / 1$ pu.

- For SoC $\leq 20 \%$, charge/discharge limits $=1 / 0$ pu.

- For $89 / 20<$ SoC $<90 / 21 \%$, charge/discharge limits vary proportionally between 0 and 1 (this is implemented for a smooth transition).

Note that since wind turbines have an inherent ES, their control algorithm is similar to that of the ES, whereas the rotor speed will set the charge/discharge limits (which are different for DFIGs and PMSG-based systems) and the $I_{o p}$ will be set by the MPPT algorithm. Due to the lack of space, wind generation systems are not considered in the paper.

MGSC control: The MGSC liaises between the MG and the grid, such that when there is a shortage of energy $\left(V_{d}<1 \mathrm{pu}\right)$, it demands energy from the grid; and when there is an excess energy $\left(V_{d}>1 \mathrm{pu}\right)$, it injects energy to the grid. This happens by controlling the $d$-component of the MGSC's current $I_{d-G}$ as shown in Fig. 2(c), which is then reflected on the grid by the NSC through controlling $V_{D C}$ by regulating the $d$-component of the NSC's current $I_{d-g}$.

The only difference between the AVR of the ES (Fig. 2(b)) and the AVR of the MGSC (Fig. 2(c)) is that there is a dead-zone of $V_{d}= \pm 0.015 \mathrm{pu}$ in the MGSC control. Doing so, reduces the energy exchanged between the MG and the grid. In other words, for $0.985<V_{d}<1.015 \mathrm{pu}$, it is the ES that balances the generation and demand, not the grid. The dead-zone, which is not a necessary part of the design, can be adjusted according to the type/performance of the ES and the required level of the involvement form the grid. The dead-zone can also be asymmetric such that it reduces/increases the imported/exported energy from/to the grid. The proposed deadzone in this paper prioritizes using the ES (over the grid) which can lead to its degradation at the cost of reducing the transmission losses through reducing the exchanged energy with the grid.

As shown in Fig. 4, a dynamic method to set $\tau_{f}$ and $\tau_{v}$ is proposed. At steady state, where frequency and voltage

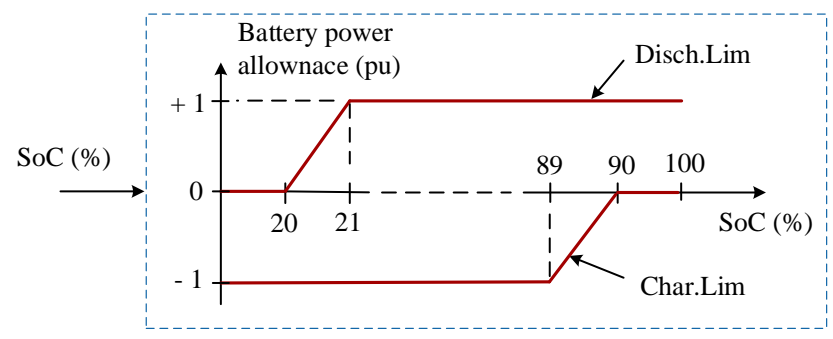

Fig. 3. Limits of $I_{d}-V_{d}$ droop for the battery unit.

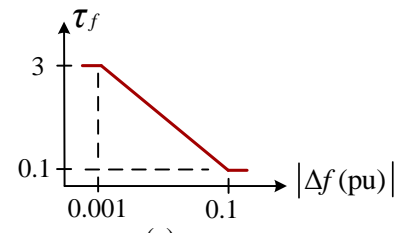

(a)

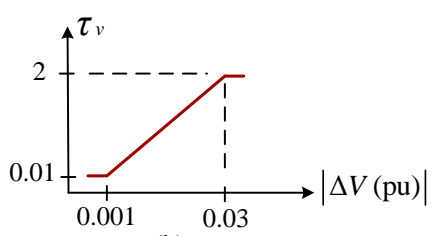

(b)
Fig. 4. Variable time-constants for low-pass filters. (a) for virtual governor. (b) for virtual AVR.

deviations $(\Delta f$ and $\Delta V)$ are small, a higher $\tau_{f}$ increases the damping factor while a small $\tau_{v}$ is required to provide a fast active power balancing/smoothing services. However, as $\Delta f$ and $\Delta V$ increase, $\tau_{f}$ reduces to increase the dynamics of the system (a faster response) while $\tau_{v}$ increases to suppress oscillations.

It is noted that the chosen voltage and frequency thresholds can be changed according to a specific Grid Code. Similarly, the 0.85 pu limit for the LVRT and the 1.02 pu generation shedding limit can vary according to a specific jurisdiction LVRT requirement and the allowed low/over-voltage limits. The other thresholds are either arbitrary or chosen empirically. For example, the dead-zone for the MGSC is absolutely arbitrary and can be changed or even removed. Similarly, the SoC limits can change for different types of battery or ES mechanisms. The thresholds of $\tau_{f}$ and $\tau_{v}$ are chosen empirically using the discussed intuition to provide a trade-off between damping and dynamics of the response.

\section{SIMULATION RESULTS}

To demonstrate the superior performance of the proposed structure and control scheme, the system shown in Fig. 1 is simulated using PSCAD/EMTDC, while different operational scenarios have been implemented. The MPPT algorithm explained in [27] is used in this paper, however, other MPPT methods are also applicable. The system parameters are given in Table I.

The load active and reactive power are modelled according to (3) and (4), respectively, which represent a combination of constant impedance, constant current, and constant power loads (ZIP model) [30]:

$$
\begin{aligned}
& P_{L}=P_{0}\left[a_{1}\left(\frac{V}{V_{0}}\right)^{2}+a_{2}\left(\frac{V}{V_{0}}\right)+a_{3}\right] \\
& Q_{L}=Q_{0}\left[a_{4}\left(\frac{V}{V_{0}}\right)^{2}+a_{5}\left(\frac{V}{V_{0}}\right)+a_{6}\right]
\end{aligned}
$$

Table I

Parameters of the simulated system

\begin{tabular}{l|c|c|c}
\hline Component & Parameter & Value & Unit \\
\hline \multirow{2}{*}{$\begin{array}{l}275 \mathrm{kV} \text { Transmission } \\
\text { Lines [28] }\end{array}$} & $R_{l}$ & 30 & $\mathrm{~m} \Omega / \mathrm{km}$ \\
\cline { 2 - 4 } & $L_{l}$ & 1 & $\mathrm{mH} / \mathrm{km}$ \\
\cline { 2 - 4 } & $C_{l}$ & 10 & $\mathrm{nF} / \mathrm{km}$ \\
\hline \multirow{2}{*}{$\begin{array}{l}\text { Power Transformers [28] } \\
\text { Cables [29] }\end{array} X_{l} \mathrm{~mm}^{2}$ XLPE } & $R_{c}$ & 0.02 & $\mathrm{pu}$ \\
\cline { 2 - 4 } & $L_{c}$ & 0.131 & $\Omega / \mathrm{km}$ \\
\cline { 2 - 4 } & $C_{c}$ & 0.38 & $\mu \mathrm{mH} / \mathrm{km}$ \\
\hline \multirow{3}{*}{ Filters } & $R_{f}$ & 0.5 & $\mathrm{~m} \Omega$ \\
\cline { 2 - 4 } & $L_{f}$ & 3.852 & $\mathrm{mH}$ \\
\cline { 2 - 4 } & $C_{f}$ & 6.577 & $\mu \mathrm{F}$ \\
\hline
\end{tabular}


where,

$V_{0}, P_{0}, Q_{0}$ : nominal values for voltage, load active power and load reactive power, respectively.

$a_{1}, a_{2}, a_{3}$ : active power factors for constant impedance, constant current and constant power loads, respectively.

$a_{4}, a_{5}, a_{6}$ : reactive power factors for constant impedance, constant current and constant power loads, respectively.

Reference [31] determined $a_{1-6}$ for different domestic, small commercial, large commercial and industrial loads. The coefficients used in this paper are the average of the coefficients of all the loads investigated in [31] i.e. $a_{1}=0.98, a_{2}=-1.19, a_{3}=$ $1.21, a_{4}=6.32, a_{5}=-10.27$ and $a_{6}=4.95$.

The following scenarios are investigated. It is noted that, due to the buffered structure of the MG, all the scenarios represent a black-start, where the nominal frequency is set and imposed by the PLL (of each DG) and the nominal voltage is implied in the pu value calculation.

\section{A. Change of load power factor}

The aim of this scenario is to demonstrate that the proposed method is a universal one and works for all types of loads. The initial purely resistive load of $(0.5+\mathrm{j} 0.0) \mathrm{pu}$ is changed over time (by $5 \%$ steps) to become purely inductive at $(0.0+\mathrm{j} 0.5) \mathrm{pu}$, leading to a change in the load power factor from 1 to 0 . Fig. 5(a) shows the power contribution of the different units, where $P_{p v 1}=0.3 \mathrm{pu}$ and $P_{p v 2}=0.2 \mathrm{pu}$ during the whole process. The difference in power due to the load change is, seamlessly, stored in the battery (see the battery power $P_{b}$ ). The resulting change in the load power factor is shown in Fig. 5(b), while Figs. 5(c) $\&$ (d) show that the load voltage magnitude and frequency are within their statutory limits [32], respectively.

\section{B. Reaching the minimum SoC limit}

In this scenario, two real solar radiation measurements (measured at Swansea University "Active Classroom" in August 2019) for a period of $70 \mathrm{~s}$ have been applied to the two PV units (see Fig. 6(a)) to investigate the system reaction against real fluctuations of the intermittent PV input power.

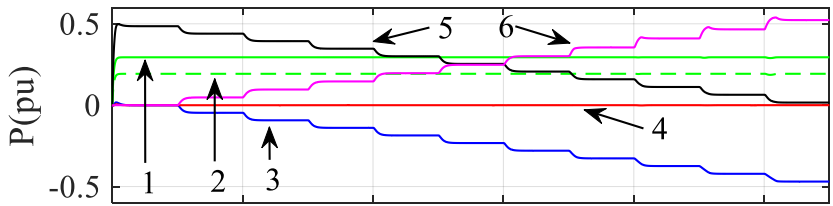

(a)

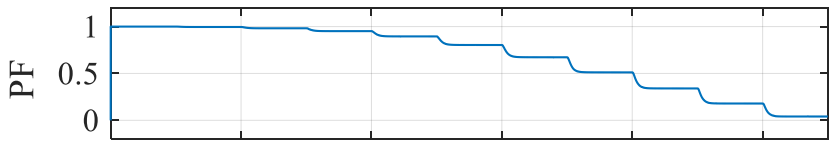

(b)
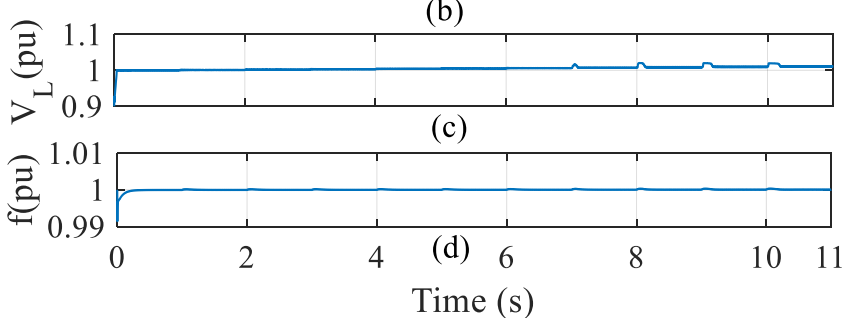

Fig. 5. Step changes in the load from pure resistive to pure inductive (a) power (pu), 1- $P_{p v 1}, 2-P_{p v 2}, 3-P_{b}, 4-P_{g}, 5-P_{L}, 6-Q_{L}$. (b) load power factor. (c) $V_{L}(\mathrm{pu})$. (d) load frequency (pu).

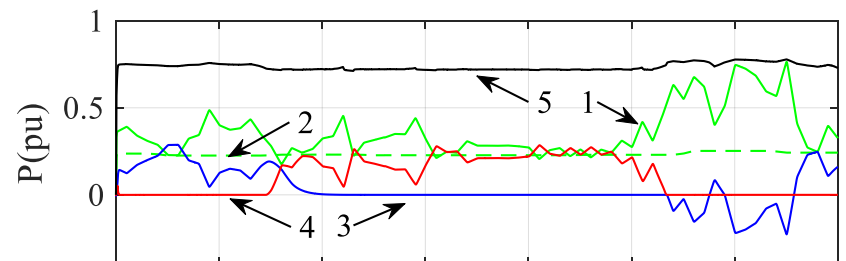

(a)

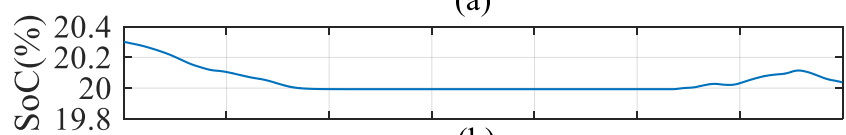

(b)

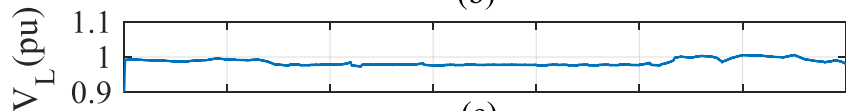

(c)

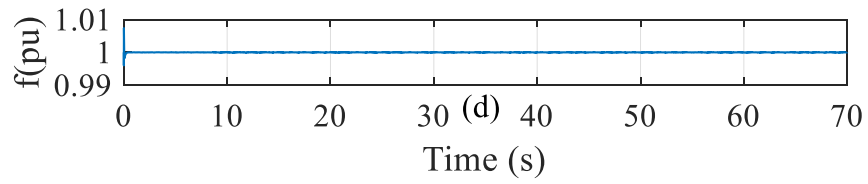

Fig. 6. Reaching the minimum $S o C$. (a) active power (pu), 1- $P_{p v 1}, 2-P_{p v 2}, 3-$ $P_{b}$, 4- $P_{g}$, 5- $P_{L}$. (b) $S o C(\%)$. (c) $V_{L}(\mathrm{pu})$. (d) load frequency (pu).

The battery SoC is set just above its minimum SoC limit of $20 \%$, while the load is assumed constant at 0.75 pu. Figs. 6(a) $\&$ (b) show the power contribution and the battery SoC, respectively. As it can be seen, until $t \approx 15 \mathrm{~s}$, while the total $P_{p v}<P_{L}$, since SoC $>20 \%$, the battery smooths out the fluctuations and balances the generation with demand (note that grid power $P_{g}=0$ ). As soon as SoC=20\%, battery power $P_{b}=0$ and grid power $P_{g}$, seamlessly, varies to supply the load. These results prove that the proposed method ensures a proper power contribution against the actual fluctuations and intermittent supply of a PV units, and show that the main grid seamlessly takes on the rest of the load when the battery SoC reaches $20 \%$. In addition, the battery charging process, seamlessly, starts again when an excess $P V$ power is available ( $t \approx 53 \mathrm{~s}$ ), stopping the main grid contribution. The load voltage and frequency are kept controlled within their statutory limits during this scenario as shown in Figs. 6(c) \& (d), respectively.

\section{Fault outside the MG, Loss of grid, Generation shedding,} Hitting Max SoC limit, Grid reconnection, Large load changes

Different sequential events are combined in this scenario as follows:

The initial settings are $P_{p v 1}=0.4 \mathrm{pu}, P_{p v 2}=0.2 \mathrm{pu}, P_{L}=0.5 \mathrm{pu}$ and the battery SoC is set slightly below its maximum limit of 90\%. Since the total $P_{p v}>P_{L}$ and SoC $<90 \%, P_{g}=0$ and SoC increases. At $\mathrm{t} \approx 7 \mathrm{~s}, \mathrm{SoC}=90 \%$, which results in the grid, seamlessly, taking on the extra energy, as seen in Figs. 7(a) \& (b). Then, a three-phase-to-ground fault takes place at $\mathrm{t}=13 \mathrm{~s}$ at the grid side ( $I_{F 1}$ in Fig. 1), followed by the opening of the circuit breakers $\mathrm{CB}_{1}$ and $\mathrm{CB}_{2}$ at $\mathrm{t}=13.14 \mathrm{~s}$, which makes $P_{g}=0$. As a result, since the ES is already fully charged, the generation shedding algorithm, seamlessly, reduces $\left(P_{p v 1}+P_{p v 2}\right)=P_{L}=0.5$ $\mathrm{pu}$. Note that when at $\mathrm{t}=16 \mathrm{~s}$ the grid is reconnected (CBs are re-closed), the MPPT algorithm is automatically restored $\left(P_{p v 1}+P_{p v 2}=0.6 \mathrm{pu}\right)$ while the grid takes on the extra energy. Then at $\mathrm{t}=20, P_{L}=\left(P_{p v 1}+P_{p v 2}\right)=0.6 \mathrm{pu}$, which makes $P_{g}=0$. As soon as $P_{L}=0.7 \mathrm{pu}>\left(P_{p v 1}+P_{p v 2}\right)$ at $\mathrm{t}=23 \mathrm{~s}$, the ES, seamlessly, 


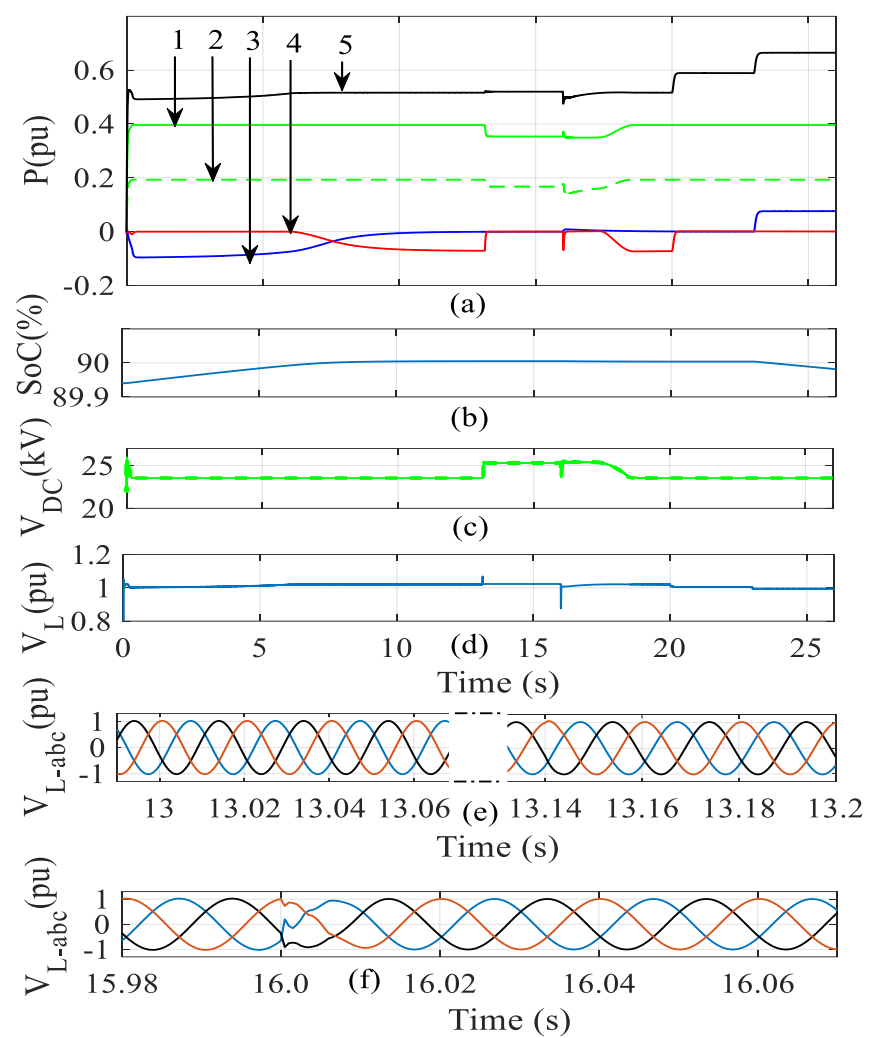

Fig. 7. Fault outside the MG, loss of grid, generation shedding, hitting max $S o C$, grid reconnection, and $10 \%$ load changes. (a) active power (pu), 1- $P_{p v 1}$, 2- $P_{p v 2}$, 3- $P_{b}, 4-P_{g}, 5-P_{L}$. (b) $S o C(\%)$. (c) $V_{p v}(\mathrm{kV})$. (d) $V_{L}$ (pu). (e) \& (f) instantaneous 3-phase load voltage zoomed-in at fault ( $\mathrm{t}=13 \mathrm{~s})$, CBs opening $(\mathrm{t}=13.14 \mathrm{~s})$ and grid reconnections ( $\mathrm{t}=16 \mathrm{~s})$ times, respectively $(\mathrm{pu})$.

supplies the shortage while $P_{g}=0$. The generation shedding process prevents PV-DC voltage from exceeding to undesired values in case of loss of the main grid, as shown in Fig. 7(c). Fig. 7(d), shows the RMS value load voltage, which is well controlled. Fig. 7(e), which shows the 3-phase load voltage zoomed-in at fault $(\mathrm{t}=13 \mathrm{~s})$ and CBs opening $(\mathrm{t}=13.14 \mathrm{~s})$ times, illustrates that the MG's voltage and frequency are not affected by the fault on the grid side. Fig. 7(f), which shows the 3-phase load voltage zoomed-in at grid reconnection time $(\mathrm{t}=16 \mathrm{~s})$, demonstrates that the frequency is well-controlled and restored in two cycles. Fig. 7(f) also shows that the phase of load voltage does not change at the time of grid reconnection.

These results demonstrate that:

- The excess energy is stored into the battery as long as it does not reach its maximum SoC, after that, it is automatically and smoothly transmitted to the main grid.

- The operation of the MG is immune from an outside fault.

- The proposed method enables a smooth and seamless transition between a non-MPPT (generation shedding) and the MPPT operations.

- The proposed method is robust against sudden large load changes (of 10\%).

\section{Fault-Ride-Through (FRT) capability}

In this scenario, symmetrical and asymmetrical faults are applied within the MG ( $I_{F 2}$ in Fig. 1) in order to examine the system FRT capability under the proposed method.

\section{1) Single-line to ground ( $S L G)$ fault}

A SLG fault takes place inside the MG ( $I_{F 2}$ in Fig.1) at $\mathrm{t}=1 \mathrm{~s}$ and lasts for $140 \mathrm{~ms} . P_{p v 1}=0.3 \mathrm{pu}, P_{p v 2}=0.2 \mathrm{pu}, P_{L}=0.6 \mathrm{pu}$, while the battery compensates for the remaining required power. Fig. 8(a) shows that the power contribution remains stable after fault clearing. Figs. 8(b) \& (c) show the $d$ - \& $q$ components of the converters' currents, respectively. It can be seen in Figs. 8(e) that voltage of the two healthy phases are kept sinusoidal during the fault period. Figs. 8(d) \& (f) show that after fault clearance the load voltage rise is under $0.3 \mathrm{pu}$, and the frequency is restored within two cycles, which is within the acceptable margins of the power system operational recommendations [33].

\section{2) Three-phase to ground fault}

In this scenario, $I_{F 2}$ (see Fig. 1) is a three-phase-to-ground fault. It takes place at $\mathrm{t}=1 \mathrm{~s}$ and lasts for $140 \mathrm{~ms}$, with the same power contribution as the SLG (previous) scenario. Fig. 9(a) shows that the power contribution remains stable after fault clearing, Figs. 9(b) \& (c) show the $d$ - \& $q$-components of the converters' currents, respectively. Figs. 9(d) \& (e) show that the voltage rise is under $0.3 \mathrm{pu}$ and the frequency is restored within two cycles, which is within the acceptable margins of the power system operational recommendations.
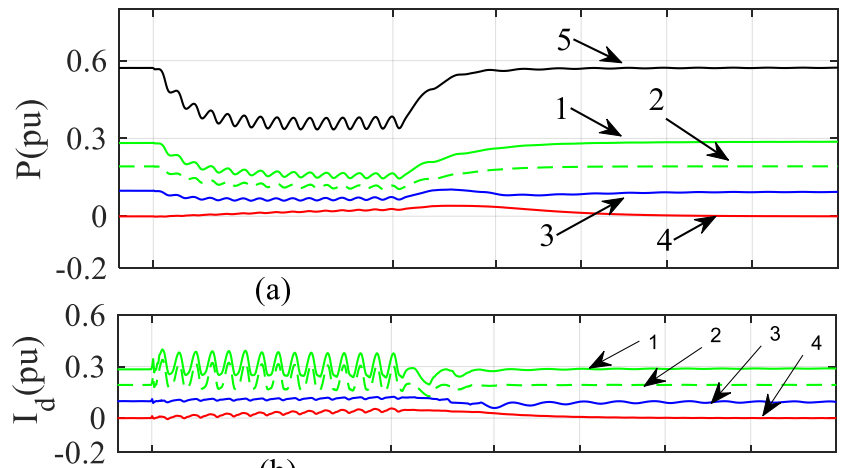

$-0.2$

(b)

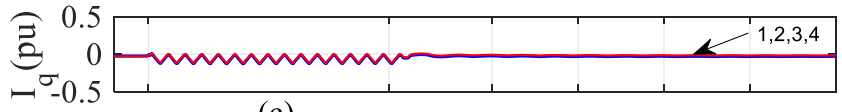

(c)
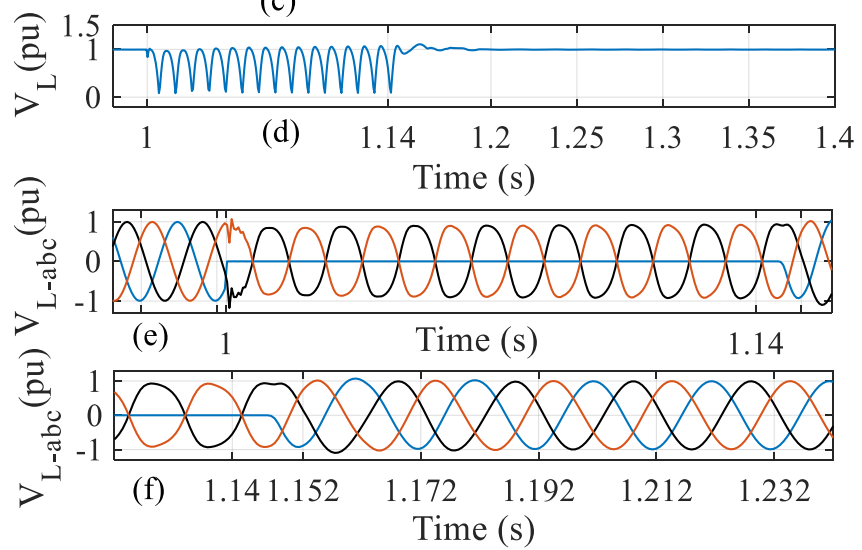

Fig. 8. SLG fault. (a) active power, 1- $P_{p v 1}, 2-P_{p v 2}, 3-P_{b}, 4-P_{g}, 5-P_{L}$. (b) $d$ components of converter currents (pu), $1-I_{d-p v 1}, 2-I_{d-p v 2}, 3-I_{d-b}, 4-I_{d-g .}$ (c) $q$ components of converter currents (pu), 1- $I_{q-p v 1}, 2-I_{q-p v 2}, 3-I_{q-b}, 4-I_{q-g}$. (d) $V_{L}$ (pu). (e) \& (f) instantaneous 3-phase load voltage zoomed-in at fault period $(\mathrm{t}=1-1.14 \mathrm{~s})$ and fault clearing $(\mathrm{t}=1.14)$ times, respectively $(\mathrm{pu})$. 


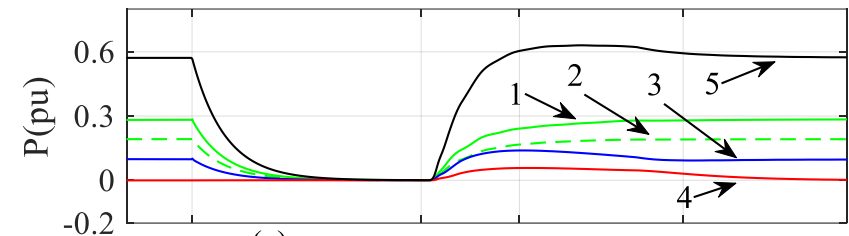

(a)

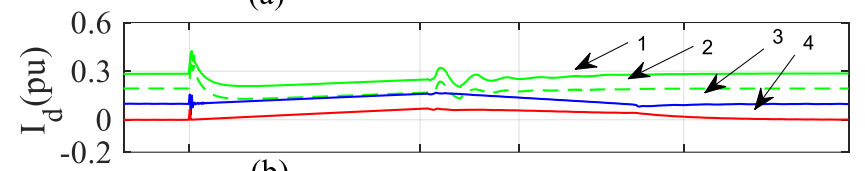

(b)

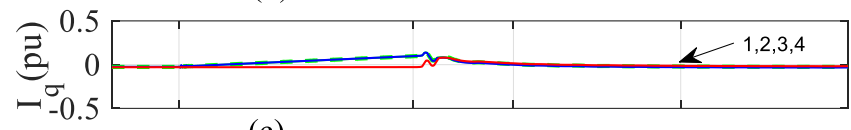

(c)
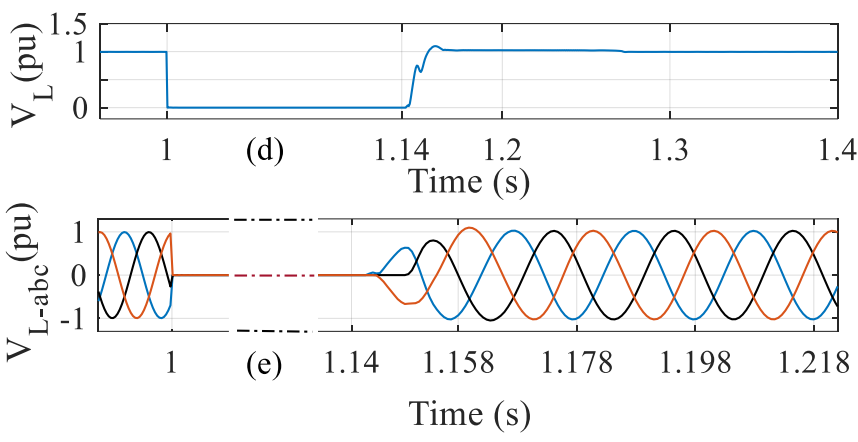

Fig. 9. Three-phase fault. (a) active power, 1- $P_{p v 1}, 2-P_{p v 2}, 3-P_{b}, 4-P_{g}, 5-P_{L}$. (b) $d$-components of converter currents (pu), 1- $I_{d-p v 1}, 2-I_{d-p v 2}, 3-I_{d-b}, 4-I_{d-g .}$. (c) $q$-components of converter currents (pu), $1-I_{q-p v 1}, 2-I_{q-p v 2}, 3-I_{q-b}, 4-I_{q-g}$. (d) $V_{L}$ (pu). (e) instantaneous 3-phase load voltage zoomed-in at fault clearing $(\mathrm{t}=1.14$ s) time (pu).

\section{E. DG outage}

This scenario investigates loss of the ES, while the load and PV power are assumed constant at $P_{L}=0.6 \mathrm{pu}$ and $P_{p v 1}+P_{p v 2}$ $=0.5 \mathrm{pu}$ (hence $P_{b}=0.1 \mathrm{pu}$ ). The ES unit, which supplies about $16.7 \%$ of the load, is disconnected at $\mathrm{t}=1 \mathrm{~s}$ to be reconnected again at $\mathrm{t}=2 \mathrm{~s}$. Fig. 10(a) shows that the grid, seamlessly, compensates for the loss in power during the ES outage time. Fig. 10(b) shows that the load voltage is kept regulated within the acceptable margins. Figs. $10(\mathrm{c}) \&(\mathrm{~d})$, which illustrate the instantaneous 3-phase load voltage zoomed-in at the ES disconnection $(\mathrm{t}=1 \mathrm{~s})$ and reconnection $(\mathrm{t}=2 \mathrm{~s})$ times, respectively, show that the frequency is restored within two cycles after the disconnection/reconnection instants.

\section{F. Central coordination for power contribution}

So far, it has been demonstrated that the localized main controller, seamlessly and with no need to a central controller, can provide balancing/smoothing capability, shed the generation, and ride through faults. Having said that, the solution is designed to enable a central control action to coordinate between units if required, e.g. to buy/sell the electrical energy between the different network operators and owners. This scenario presents an energy exchange between the ES unit and the main grid, while $\left(P_{p v 1}+P_{p v 2}\right)=P_{L}=0.5 \mathrm{pu}$. As shown in Fig. 11(a):

- Initially, there is no energy exchange between the grid and the MG, as the load is covered by the PV units, while no central control commands are applied i.e. $I_{o p}=0$.

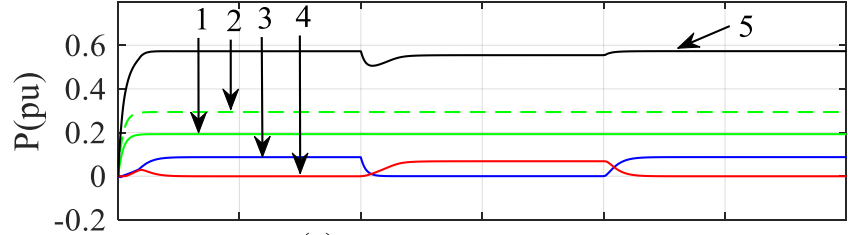

(a)
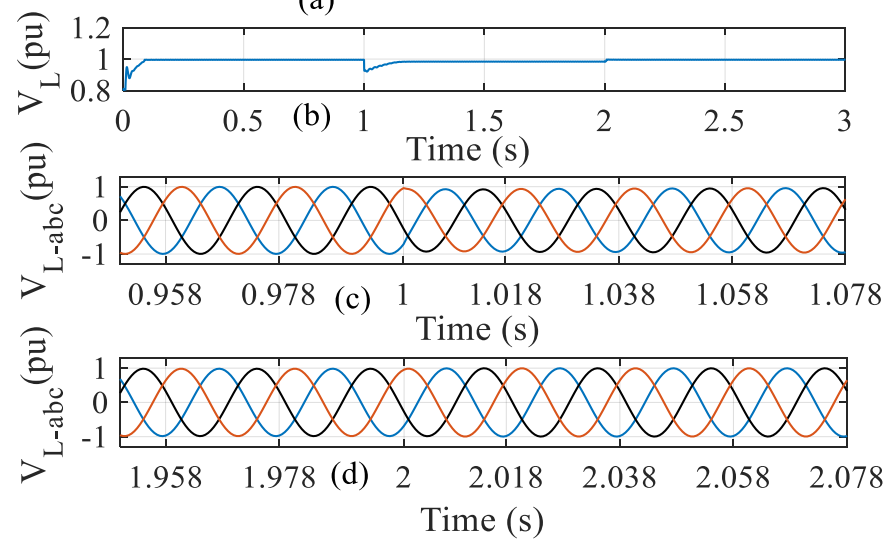

Fig. 10. ES unit outage. (a) power (pu), 1- $P_{p v 1}, 2-P_{p v 2}, 3-P_{b}, 4-P_{g}, 5-P_{L}$. (b) $V_{L}$ (pu). (c) \& (d) instantaneous 3-phase load voltage zoomed-in at the ES disconnection ( $\mathrm{t}=1 \mathrm{~s})$ and reconnection $(\mathrm{t}=2 \mathrm{~s})$ times, respectively $(\mathrm{pu})$.

- At $\mathrm{t}=2 \mathrm{~s}$, a central command is applied for a time duration of $5 \mathrm{~s}$, through the component $I_{o p}=+/-0.1 \mathrm{pu}$, to the control systems of the ES/MGSC units (see Fig. 2(b) \& (c)). Doing so, leads to charge the battery, demanding energy from the main grid.

- At $\mathrm{t}=7 \mathrm{~s}, I_{o p}=0$ for a time duration of $2 \mathrm{~s}$ (for both ES and MGSC), stopping the energy exchange process.

- $\mathrm{Ag} \mathrm{t}=9 \mathrm{~s}, I_{o p}=-/+0.1 \mathrm{pu}$, for a time duration of $5 \mathrm{~s}$, is applied to the control systems of the ES/MGSC units, leading to export energy from the ES to the main grid.

Fig. 11(b) illustrates the battery SoC during this process. Figs. 11(c) \& (d) show that the load voltage and frequency are maintained within their acceptable values, respectively.

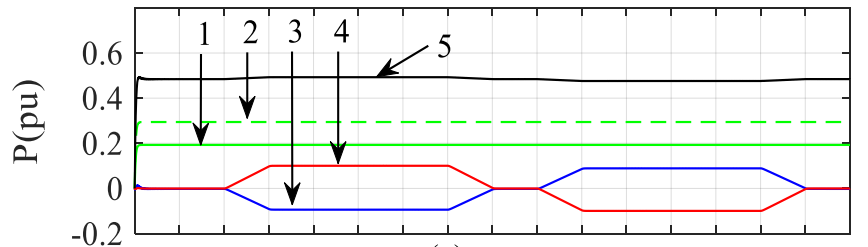

(a)
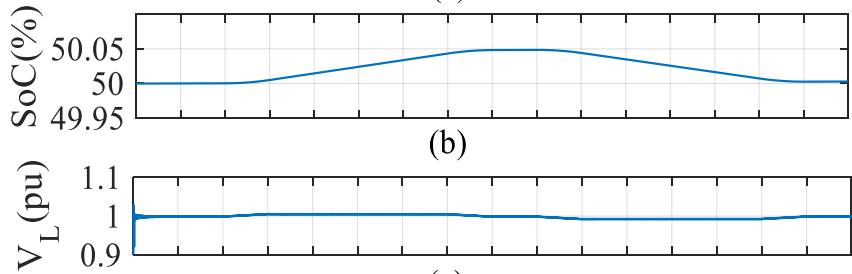

(c)

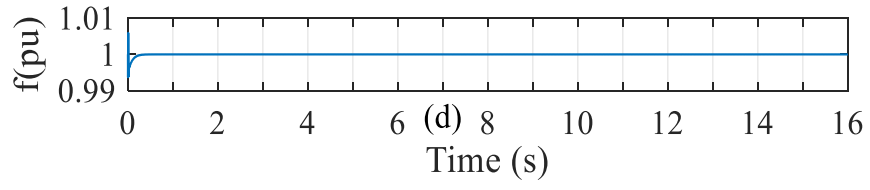

Fig. 11. Central power coordination. (a) active power, 1- $P_{p v 1}, 2-P_{p v 2}, 3-P_{b}, 4-$ $P_{g}, 5-P_{L}$. (b) $\operatorname{SoC}(\%)$. (c) $V_{L}(\mathrm{pu})$. (d) load frequency (pu). 


\section{CONCLUSION}

This paper proposed a new structure alongside a new control system for MGs that enables a complete seamless operation of the MGs for all operational modes/scenarios and for all types of loads. The proposed structure involves decoupling the MG from the grid using a set of AC/DC/AC converters. Such a "buffered" structure enables faster voltage and frequency control within a MG by separating the dynamics of the MG from the slow dynamics of the grid's SGs. This structure also protects the MGs from the external faults and the low frequency oscillations (e.g. inter-area oscillations) that exist in the grid or can be created due to the interactions between the DERs and the SGs.

The dead-zone proposed in controlling the MGSC delays the involvement of the grid in the energy management of the MG (until the ES is fully charged/discharged), which reduces the exchanged energy and the transmission losses. Moreover, the proposed method protects the ES from over charging/discharging through ensuring a smooth and seamless transition of power to/from the main grid. The generation shedding algorithm ensures the operation continuity in islanded mode where an excess of energy is available in the MG, preventing the DC voltage of the DER from exceeding to undesirable values. While the primary control enables an absolute communication-free voltage and frequency control, power smoothing/balancing and fault ride-through, it is possible to override the primary controller through a central control to coordinate between units. To demonstrate the superior performance of the proposed system, PSCAD/EMTDC was used to simulate extensive scenarios including: variation of loads from pure restive to pure inductive, fully charged/discharged ES, generation shedding, large load changes, DERs outage and fault ride-through for faults on both the grid- and the MG-sides.

Although the proposed structure incurs the extra costs of the $\mathrm{AC} / \mathrm{DC} / \mathrm{AC}$ converters, considering the advantages (e.g. protection from the external faults, simple and communicationfree operation), it seems that the extra costs is justifiable (at least for sensitive/important loads such as national servers). However, this paper does not claim that a Cost-Benefit Analysis is performed, which indeed can vary for each case.

\section{REFERENCES}

[1] The National Archives. Climate Change Act 2008. HM Government. Surrey, UK. [08/01/2020]. Available:

http://www.legislation.gov.uk/ukpga/2008/27/contents.

[2] National Grid. (2019, July). Future Energy Scenarios. NationalGridESO. Warwick, UK. [08/01/2020], Available: http://fes.nationalgrid.com/media/1409/fes-2019.pdf.

[3] A. Hirsch, Y. Parag and J. Guerrero, "Microgrids: A review of technologies, key drivers, and outstanding issues," Renewable and Sustainable Energy Reviews," vol. 90, pp. 402-411, 2018.

[4] N. Hatziargyriou, H. Asano, R. Iravani and C. Marnay, "Microgrids," IEEE Power and Energy Magazine, vol. 5, no. 4, pp. 78-94, 2007.

[5] R. Lasseter, A. Akhil, C. Marany, J. Stephens, J. Dagle, R. Guttomson, A. S. Meliopoulous, R. Yinger and J. Eto. (2002, April). Integration of distributed energy resources. The CERTS Microgrid Concept. [22/01/2020]. Available:

https://escholarship.org/uc/item/9w88z7z1.

[6] M. Fazeli, G. M. Asher, C. Klumpner and L. Yao, "Novel Integration of DFIG-Based Wind Generators Within Microgrids," IEEE Trans. Energy Convers., vol. 26, (3), pp. 840-850, 2011.

[7] M. Fazeli, G. M. Asher, C. Klumpner, L. Yao and M. Bazargan, "Novel Integration of Wind Generator-Energy Storage Systems Within
Microgrids," IEEE Transactions on Smart Grid, vol. 3, (2), pp. 728-737, 2012.

[8] H. Almasalma, J. Engels and G. Deconinck, "Peer-to-Peer Control of Microgrids," in the 8th IEEE Benelux Young researchers symposium in Electrical Power Engineering, Eindhoven, Netherland, 2016.

[9] P. Piagi and R. H. Lasseter, "Autonomous control of microgrids", Power \& Energy Society, IEEE General Meeting, Montreal, Canada, 2006.

[10] R. Schollmeier, "A definition of peer-to-peer networking for the classification of peer-to-peer architectures and applications", Peer-toPeer computing, Linkoping, Sweden, 2001, pp. 101-102.

[11] Y. Zhu, F. Zhuo and H. Shi, "Accurate power sharing strategy for complex microgrid based on droop control method", IEEE ECCE Asia Downunder, Melbourne, Australia, 2013, pp. 344-350.

[12] R. Majumder, B. Chaudhuri, A. Ghosh, R. Majumder, G. Ledwich and F. Zare, "Improvement of Stability and Load Sharing in an Autonomous Microgrid Using Supplementary Droop Control Loop," IEEE Transactions on Power Systems, vol. 25, no. 2, pp. 796-808, 2010.

[13] R. Majumder, A. Ghosh, G. Ledwich and F. Zare, "Load sharing and power quality enhanced operation of a distributed microgrid," IET Renewable Power Generation, vol. 3, no. 2, pp. 109-119, 2009.

[14] A. M. Egwebe, M. Fazeli, P. Igic and P. M. Holland, "Implementation and Stability Study of Dynamic Droop in Islanded Microgrids," IEEE Trans. Energy Convers., vol. 31, no.3, pp. 821-832, 2016.

[15] H. Mahmood, D. Michaelson and J. Jiang, "A Power Management Strategy for PV/Battery Hybrid Systems in Islanded Microgrids," IEEE Journal of Emerging and Selected Topics in Power Electronics, vol. 2, no. 4, pp. 870-882, 2014.

[16] Y. Karimi, H. Oraee, M. S. Golsorkhi and J. M. Guerrero, "Decentralized Method for Load Sharing and Power Management in a PV/Battery Hybrid Source Islandd Microgrid," IEEE Transactions on Power Electronics, vol. 32, no. 5, pp. 3525-3535, 2017

[17] Y. Zhang, R. A. Dougal and H. Zheng, "Tieline Reconnection of Microgrids Using Controllable Variable Reactors," IEEE Transactions on Industrial Applications, vol. 50, no. 4, pp. 2798-2806, 2014.

[18] F. Tang, J. M. Guerrero, J. C. Vasques, D. Wu and L. Meng, "Distributed Active Synchronization Strategy for Microgrid Seamless Reconnection to the Grid under Unbalance and Harmonic Distortion," IEEE Transactions on Smart Grid, vol. 6, no.6, pp. 2757-2769, 2015.

[19] D. Shi, X. Chen, Z. Wang, X. Zhang, Z. Yu, X. Wang and D. Bian, "A Distributed Cooperative Control Framework for Synchronized Reconnection of a Multi-Bus Microgrid," IEEE Transactions on Smart Grid, vol. 9, no. 6, pp. 6646-6655, 2018.

[20] M. Fazeli and P. Holland, "Universal and Seamless Control of Distributed Resources-Energy Storage for All Operational Scenarios of Microgrids," IEEE Transactions on Energy Conversion. vol. 32, no. 3, pp. 963-973, 2017.

[21] S. M. Ashabani and Y. A. I. Mohamed, "New Family of Microgrid Control and Management Strategies in Smart Distribution Grids-Analysis, Comparison and Testing," IEEE Transactions on Power Systems, vol. 29, no. 5, pp. 2257-2269, 2014.

[22] R. Perez-Ibacache, A. Yazdani, C. Silva and J. C. Aguero, "Decentralized Unified Control for Inverter-based AC Microgrids Subject to Voltage Constraints," IEEE Access, vol. 7, pp. 157318-157329, 2019.

[23] C. Wang, B. Liang and J. He, "An Enhanced Power Regulation and Seamless Operation Mode Transfer Control through Cooperative DualInterfacing Converters," IEEE Transactions on Smart Grid, vol. 9, (6), pp. 5576-5587, 2018.

[24] A. Kirakosyan, E. El-Saadany, M. S. El Moursi, A. H. Yazdavar and A. Al-Durra, "Communication-Free Current Sharing Control Strategy for DC Microgrids and its Application for AC/DC Hybrid Microgrids," IEEE Transactions on Power Systems, vol. 25, no. 1, pp. 140-151, 2019.

[25] J. Quintero, V. Vittal, G. T. Heydt and H. Zhang, "The Impact of Increased Penetration of Converter Control-Based Generators on Power System Modes of Oscillation," IEEE Transactions on Power Systems, vol. 29, no. 5, pp. 2248-2256, 2014.

[26] Qing-Chang Zhong and Y. Zeng, "Universal Droop Control of Inverters With Different Types of Output Impedance," IEEE Access, vol. 4, pp. 702-712, 2016.

[27] M. Fazeli, P. Igic, P. Holland R. Lewis and Z. Zhou, "Novel maximum power point tracking with classical cascaded voltage and current loops for photovoltaic systems," in IET Conference on Renewable Power Generation. Edinburgh, UK. 2011. pp. 24-29.

[28] National Grid. (2011, November). Transmission System Data: GB National Grid, UK. [10/05/2020], Available: http://gnu.ets.kth.se/ nt/elecpow/gb ng par/gb trans ng. 
[29] Torne \& Derrick International. [2020]. 11kV Cables XLPE 3 Core 185sqmm, BS6622 Nexans. [18/05/2020] Available:

https://www.powerandcables.com/product/product-category/11kvcables-xlpe-3-core-185sqmm-bs6622-nexans/.

[30] J. Machowski, J. W. Bialek, K. R. Bumby, "Power System Loads" in "Power System Dynamics Stability and Control. 2nd ed. . John Wily \& Sons, Ltd. Wiltshire, UK, 2008. pp. 104-113.

[31] A. Bokhari, A. Alkan, R. Dogan, M. Diaz-Aguiló, F. de León, D. Czarkowski, Z. Zabar, L. Birenbaum, A. Noel and R. E. Uosef, "Experimental Determination of the ZIP Coefficients for Modern Residential, Commercial, and Industrial Loads," IEEE Transactions on Power Delivery, vol. 29, no. 3, pp. 1372-1381, 2014.

[32] Voltage Characteristics of Electricity Supplied by Public Distribution Systems. BS EN 50160, 2000.

[33] National Grid. (2019, July). Stability Pathfinder RFI: Technical Performance and Assessment Criteria. NationalGridESO. Warwick, UK. [28/02/2020]. Downloadable from:

https://www.nationalgrideso.com/publications/network-options assessment-noa/network-development-roadmap. 